FEASIBILITY TESTS OF A COMMERCIAL

RADIOGRAPHIC LINAC FOH NUCLEAR

IIATTERTALS ASSAY ${ }^{+}$

\title{
Conf- $710617--13$
}

MESTER

\author{
L. A. Kull and G. M. Reynolds \\ JRB Associates, Inc., La Jolla, California
}

E. Wattacamps
Gesellshaft Fuer Kernforschung, Karlsruhe, Germany $357^{10^{\circ}}$

E. Weinstock

Safeguards Technical Services Organization,

Brookhaven National Laboratory, Brookhaven, Long Island

\section{INTRODUCTION}

Tests have been made on a variable energy, standing wave radiographic electron linac, * under the direction of the Safeguards Technical Services Organization at the Brookhaven National Laboratory, to determine the feasibility of using commercially available electron linacs for non-destructive assays of special nuclear materials. Assay techniques similar to those developed in the Safeguards research and development program at Gulf Radiation Technology and sponsored by the AEC Office of Safeguards and Materials Management, were used to test the linac parameters that are important for ron-destructive measurements. Some of the parameters are:

1. stability of the electron energy spectrum during a series of assays,

2. reproducibility of the electron energy spectrum after changing back and forth between different electron energies,

3. reproducibility of the electron energy spestrum after power shut-down, and.

4. general performance characteristics of the accelerator during the tests.

\footnotetext{
* The tests were made for the Safeguards Technical Services Organization of Brookhaven National Laboratory in cooperation with personnel from the accelerator division of Varian Associates, Palo Alto, California.

Manufactured by Varian Associates, Palo Alto, California.
} 
To check the stability and reproducibility of the electron energy spectrum, the yields of prompt and delaved neutrons from a fissionable target $\left(U^{238}\right)$ were measured for a series of runs at two electron energies. The electron radiation was converted to a forward directed bremsstrahlung beam by a platinum converter. Prompt photoneutrons, prompt photofission neutrons, and delayed photofission neutrons were produced by the brensstrahlung beam incident on the $\mathrm{U}^{238}$ target. The neutron yields rise sharply with increasing electron energy for 6 to $9 \mathrm{MeV}$ electrons, which is the energy region covered in these iests. Therefore, if the electron spectrum of the linac was unstable, or was not reproduced after changes in the accelerator's operating modes, it would be readily observed as a change in the measured neutron yield.

The measurements were made on a modified version of the Linatron 1500 in a test celi at the Varian facilities in Palo Alto, California. Figures 1 and 2 show views of the linac X-ray head and control panel, respectively. An energy analyzing magnet is not included as a part of the linac system, making the requirements for stability and reproducibility of the eleciron spectrum very stringent. The tests were conducted in this mannex to check a previcusly reported contention that the side-ccupled, standing wave linac design offered the possibility of sufficient energy stability, without an analyzing magnet system, for use in Safeguards assay applications. (1)

The tests were a cooperative effort which involved personnei from JRB Associates and from the Technical Services Organization at Brookhaven, representatives from the Gesellshaft Fuer Kernforschung, Karlsrulie, Germany, the technical staff at Varian Associates, and the nuclear engineering staff at Stanford University.

\section{EXPERIMENTAL PFOCEDURES}

The positions of the target and neutron detecior with respect to the linac are shown in Fig. 3. The geometry and distances were similar to those that would be encountered in an actual Safeguards application. $A$ lead and polyethylene shield between the $\mathrm{BF}_{3}$ slab detector and the electron-tobremsstrahlung converter and beam collimators reduced the effect of gamma-flash and background photoneutrons proriced in the converter and collimators. 
Fig. 1 Waveguide and R assemibly - Linatron 1500.

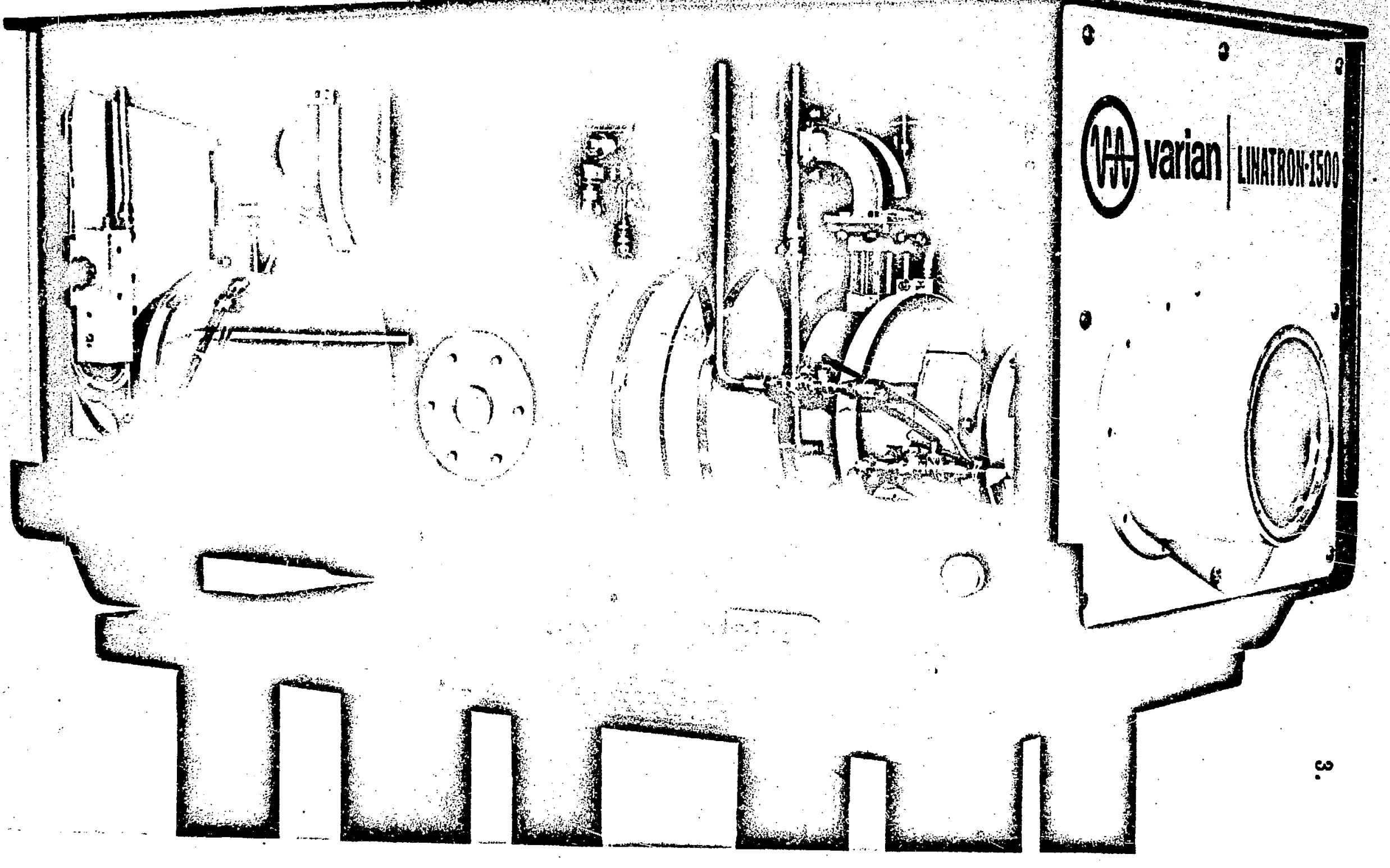


Fig. $z$ Control panel - Linatron 1500.

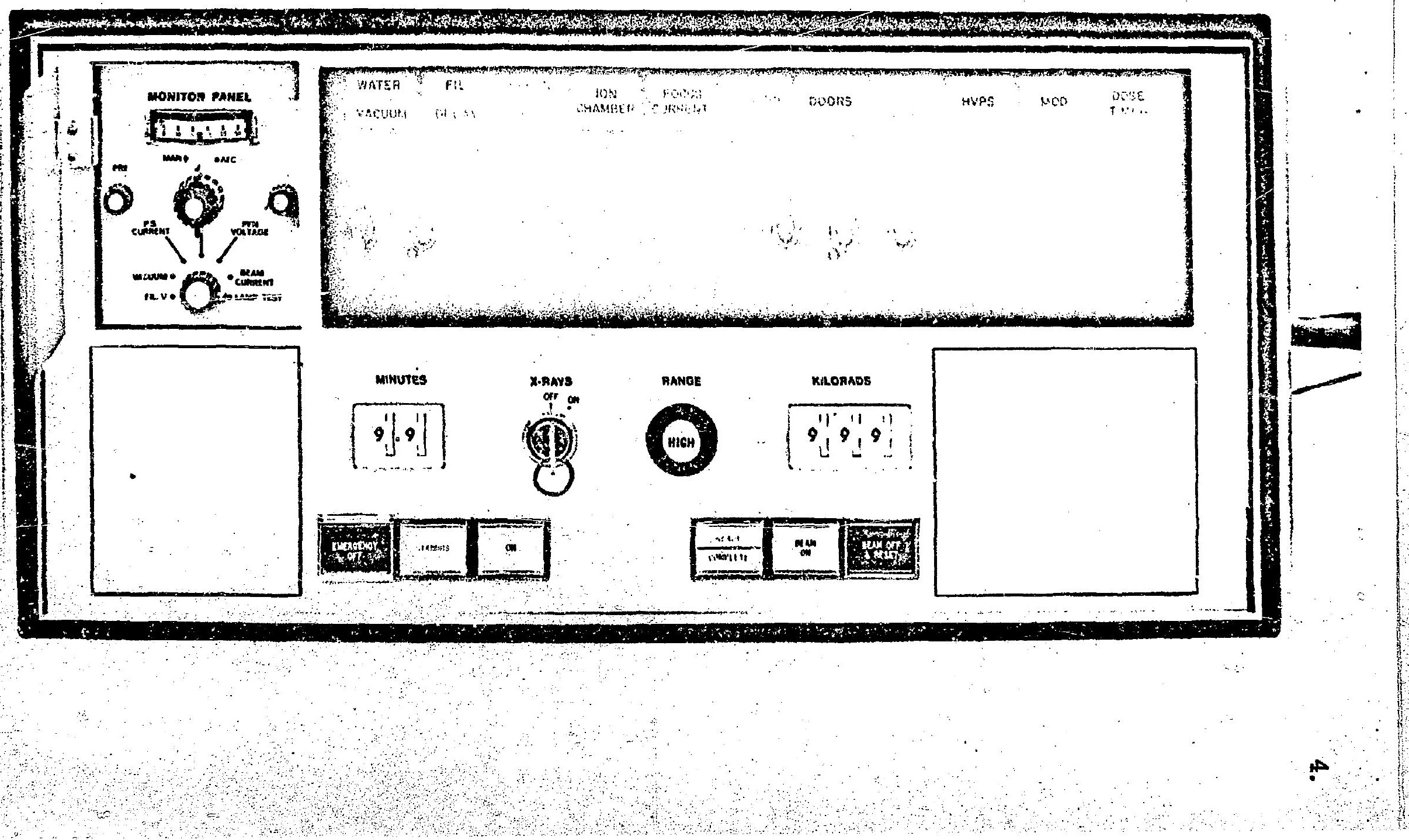




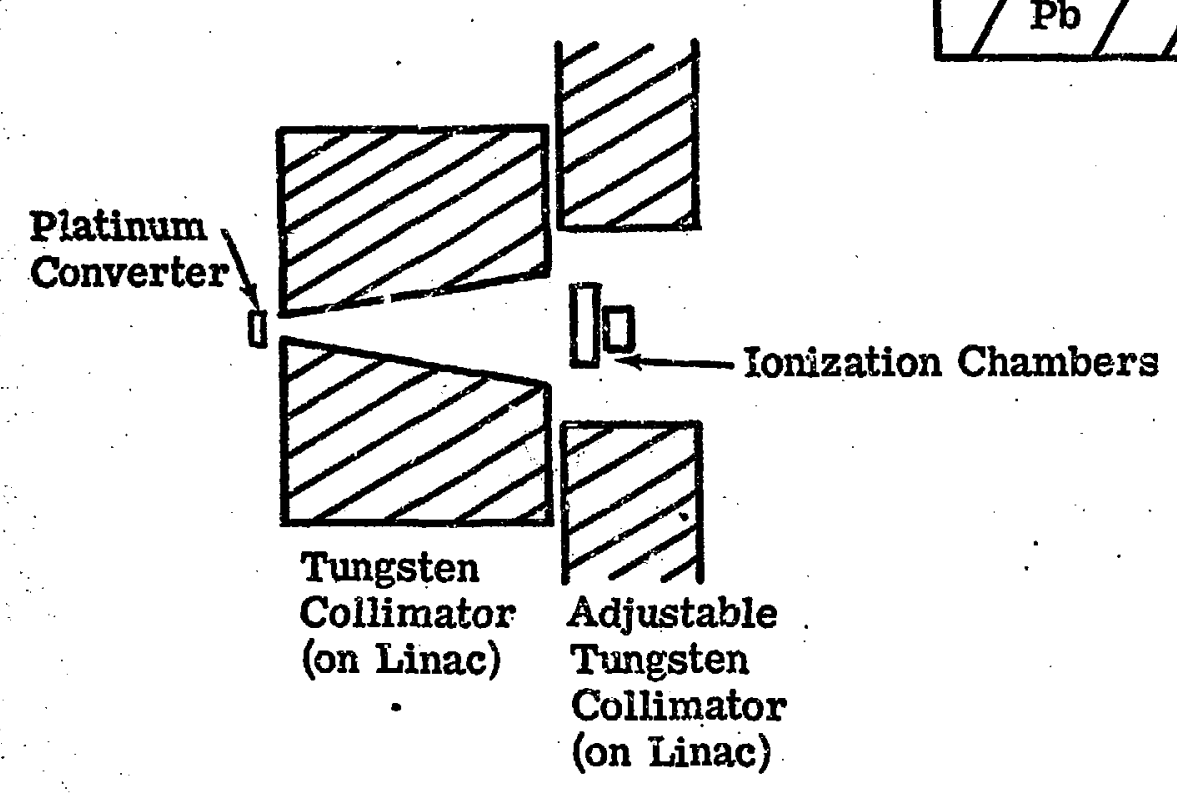

Approximate Scale
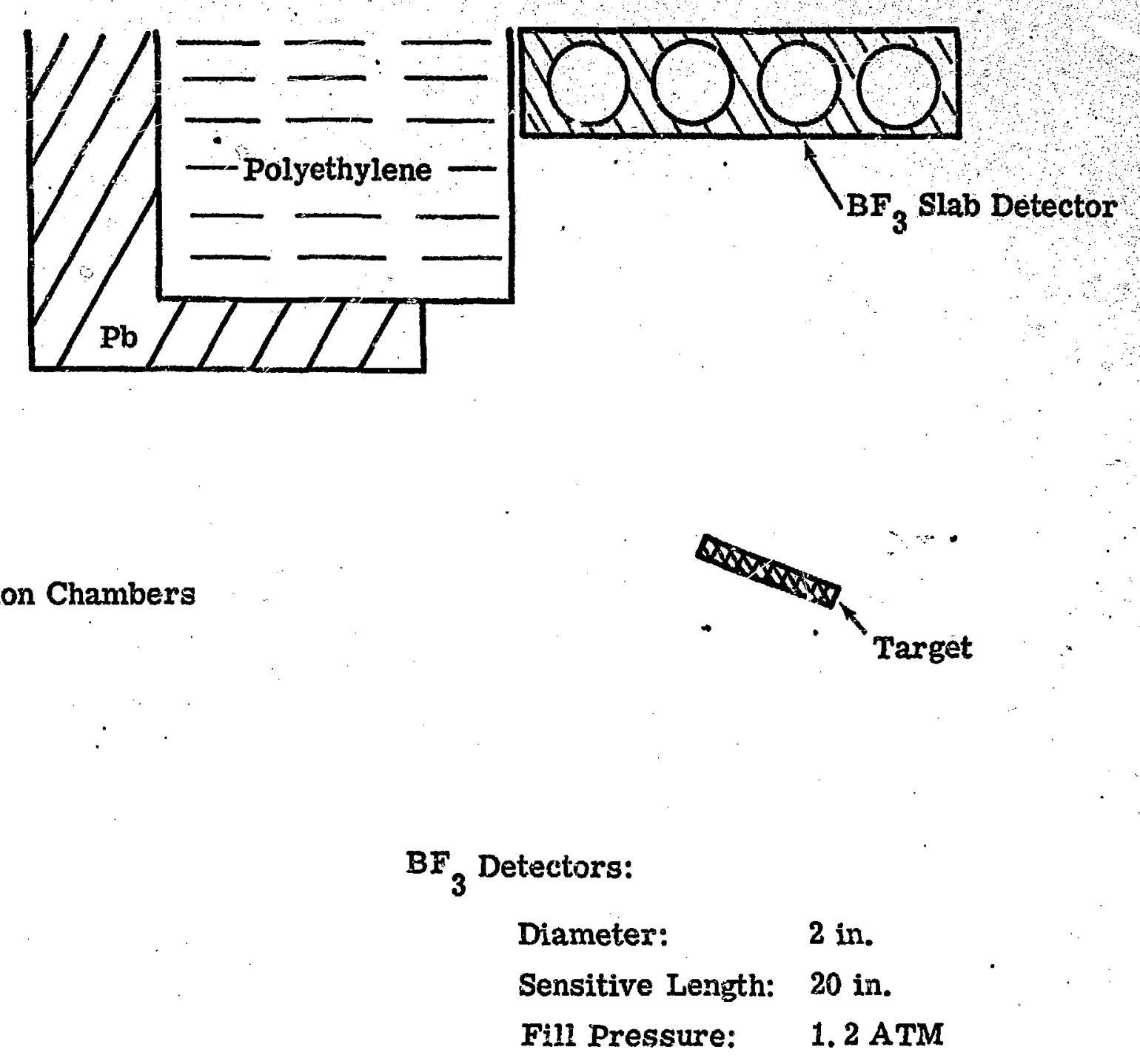

6 in.

Fig. 3. Linac and detector geometry. 
The neutron detector consisted of $4 \mathrm{BF}_{3}$ tubes (2" diameter, $20^{\prime \prime}$ sensitive length) in a polyethylene moderator. A thin layer of cadmium $\left(0.040^{\prime \prime}\right)$ surrounded the polyethylene to reduce the effects of low-energy room-return neutrons on the yieid of delayed fission neutrons. The slab detectox contained a preamp and pulse shaping network of short timeconstant, to decrease recovery time* from the gamma-flash over-load pulse. The recovery time for this system was typically around $25 \mu \mathrm{sec}$.

Details of the electronics are shown in Fig. 4. Initially a TMC multichannel analyzer was used to check the pulse height distribution of the slab detector, to set the level of the discriminator, and to measure the characteristic die-away time of the detector. The detector decay constant was found to be $77 \mu \mathrm{sec}$, which is typical for this kind of detector. In taking the data, two scalers were used, one to count total neutrons (prompt and delayed) and one to count only delayed neutrons. Counting during the gamma-flash overload was prevented by the linear gate.

For these tests the linac was set to run at two different electron energies, around 6 and $8 \mathrm{MeV}$. The beam parameters are given in the following table.*

\section{TABLE 1}

BEAM PARAMETERS

\begin{tabular}{|c|c|c|c|c|}
\hline & $\begin{array}{c}\text { Pulse rate } \\
\text { (pulses/sec) }\end{array}$ & $\begin{array}{c}\text { Puise width } \\
\text { (usec) }\end{array}$ & $\begin{array}{c}\text { Peak current } \\
(\mathrm{ma})\end{array}$ & $\begin{array}{c}\text { Average current } \\
(\mu \mathrm{a})\end{array}$ \\
\hline "6" MeV & 105 & 3 & 6 & 2.0 \\
"8" MeV & 105 & 3 & 1 & 0.37 \\
\hline
\end{tabular}

* The recovery time is defined as the length of time necessary for. the detector to attain its normal operating condition after being exposed to the intense bremsstrahlung "gamma-flash".

A more complete set of specifications for this accelerator is included in Appendix 1 . 


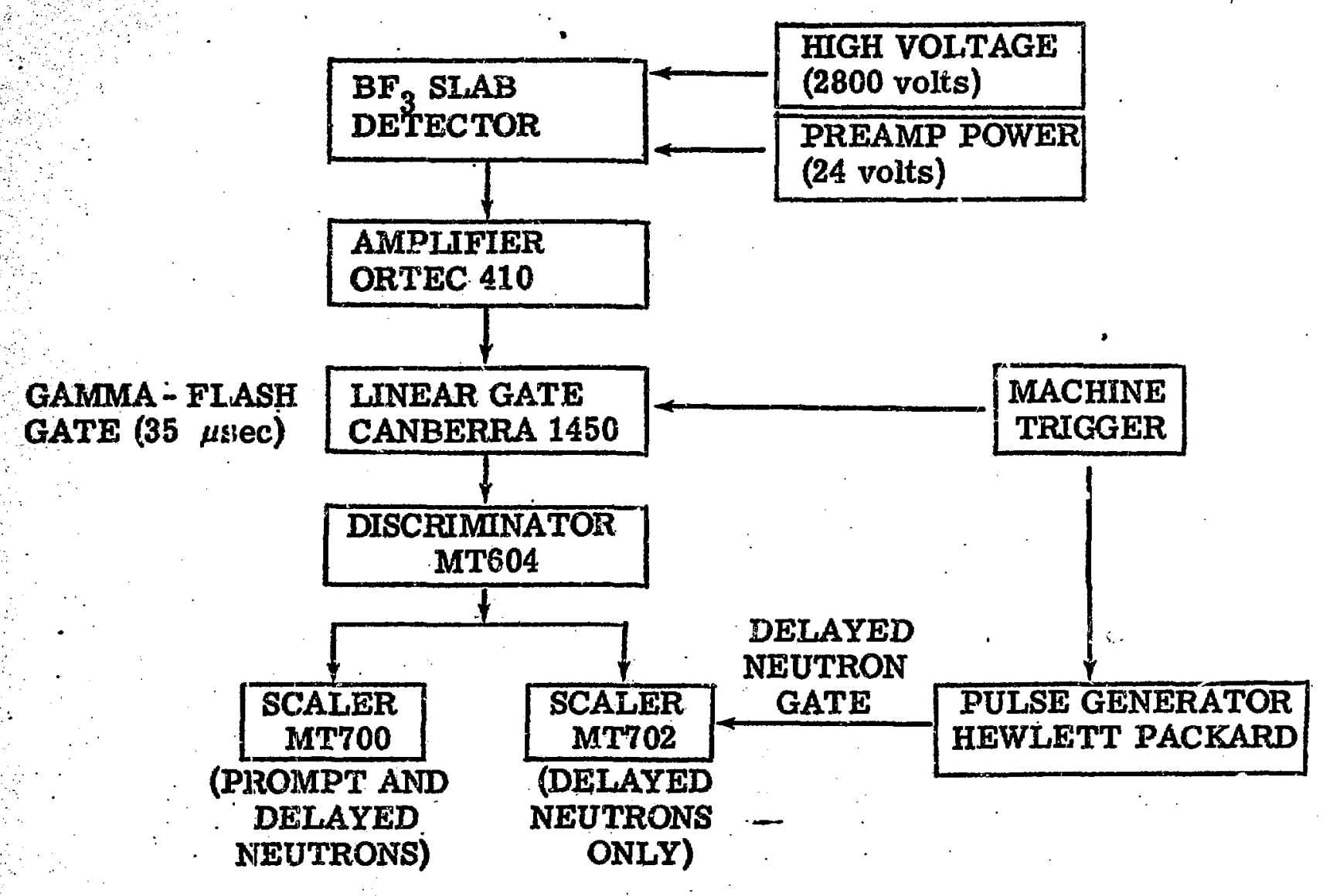

\section{Linear Gate:}

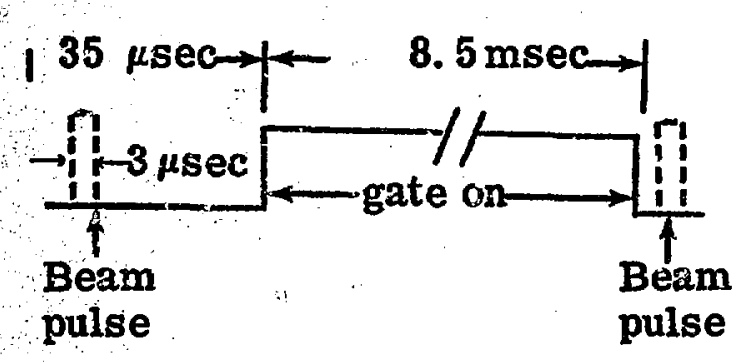

Delayed Neutron Gate:

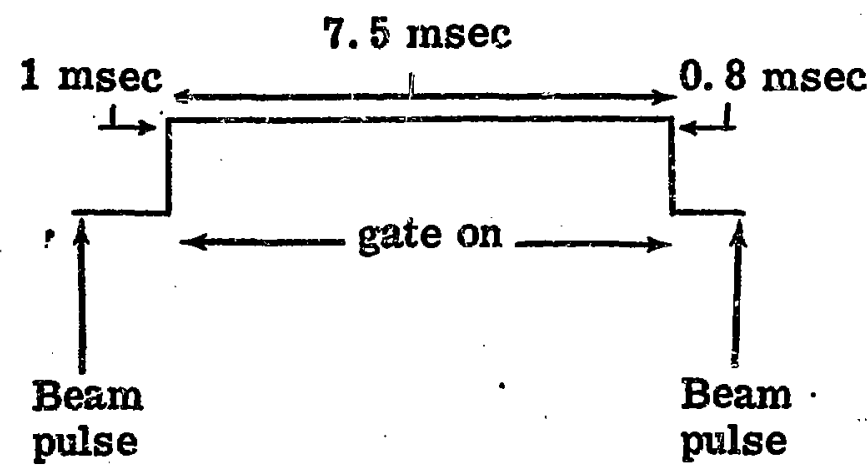

Fig. 4 Detector Electronics 
Normally the beam currents from the Varian linacs are fixed for each electron energy. The currents can be set at desired values before final installation of the machine by (a) adjusting a voltage divicier on the gun to vary the injected current, and (b) changing the power to the magnetron by adjustments to the modulator pulse forming network. The beam currents used in these tests were selected to give reasonable counting rates from the fissioniole material samples.

The peak electron current on the platinum converter was read off a scope. To calibrate the neutron yields, a pair of sealed ion chambers in the center of the bremsstrahlung beam were used. The total integrated gamma-ray dose in the ion chambers was measured for each run.

The uranium target consisted o: a piece of depleted uranium, $10 \mathrm{~cm} \mathrm{x}$ $15.2 \mathrm{~cm} \times 3.3 \mathrm{~cm}$. A copper block similar in size to the U238 target was used to obtain the background yields of prompt and delayed neutrons. The copper acts as a gamma scatterer withcut producing any neutrons (the $(\gamma, n)$ threshold for copper is $9.9 \mathrm{MeV}$, which is higher than the electron energies used in the test). The delayed neutrons measured with the copper target in place arise from prompt photoneutrons which have been multiply scattered from surrounding material.

\section{RESULTS AND ANALYSIS}

Approximately one hundred data runs were obtained for targets of $\mathrm{U}^{238}$ and copper. The total gamma dose, the time, and the prompt and delayed neutron yields were measured for each run. The energies are shown in quotation marks since, without analyzing magnets, they are rot known precisely. 'The following methods were used to get an estimate of the artuai electron energies:

1. the range of the electrons was measured.

2. the ratios of the prompt to delayed neutrons were measured and compared with previous measurements made as a function of electron energy $(2,3)$.

3. the absolute yield of delayed neutrons vs. electron energy was measured and compared with existing data. 
The prompt and delayed yields must be corrected for effects of the gamma-flash gate and delayed neutron gate. About $30 \%$ of the prompt neutrons are not counted due to the $35 \mu \mathrm{sec}$ wait for the gamma-flash recovery. After each beam burst the prompt neutron counting dies away in the slab detector accoding to $e^{-\lambda t}$ where $\lambda^{-1}=77 \mu$ sec. If $t_{0}$ is the width of the gamma-flash gate, then the ratio of prompt neutron counts lost during the gate $\left(\mathrm{N}_{0}\right)$, to the prompt neutrons counted after the gate $\left(N_{1}\right)$ is given by

$$
\frac{N_{0}}{N_{1}}=\frac{\left(1-e^{-\lambda t_{0}}\right)}{e^{-\lambda t_{0}}}
$$

For $t_{0}=35 \mu \mathrm{sec}$, the ratio $\mathrm{N}_{0} / \mathrm{N}_{1}=0.567$. The total prompt yield $(\mathrm{N})$ can therefore be reiated to the measured prompt yield $\left(\mathrm{N}_{1}\right)$ by

$$
\mathrm{N}=\mathrm{N}_{0}+\mathrm{N}_{1}=\mathrm{N}_{1}\left(1+\frac{\mathrm{N}_{0}}{\mathrm{~N}_{1}}\right)=1.567 \mathrm{~N}_{1}
$$

During a run the delayed neutroin production rate is essentialiy constant. Since the delayed neutron scaler is on only a fraction of the time, a correction factor must also be applied to the delayed yield. The gating times in Fig. 4 and two runs with a constant neutron source (PuBe source) indicated that the actual delayed neutron yield is 1.24 times the obser ved yield.

Using the corrected yields and previous data on prompt/delayed neutron yields at various electron energies, an estimate of the electron energies obtrined are shown in Table 2.

The prompt neutron yieid could not be obtained at the higher energy due to excessive count rate losses from high backgrounds. However, comparing the relative yields of delayed neutrons at the two energies with those from previous measurements, $(2,3)$ does allow an estimate of the higher energy. The estimated electron energies from the range measuraments, performed by the Varian staff, are also shown in Table 2. Finaliy, the delayed neutron yields have been calculated from the measured 
TABLE 2.

. ELECTRON ENERGY ESTIMATES

\begin{tabular}{|c|c|c|c|c|}
\hline $\begin{array}{l}\text { Electron } \\
\text { Energy } \\
\text { (MeV) }\end{array}$ & $\underset{\substack{\mathrm{U}^{238} \\
\text { Ratio }}}{\text { Prompt/Delayed }}$ & $\begin{array}{l}\text { Electron Energy } \\
\text { (using Prompt/ } \\
\text { Delayed Ratio) }\end{array}$ & $\begin{array}{c}\text { Electron } \\
\text { Energy } \\
\text { (Range Meas.) }\end{array}$ & $\begin{array}{l}\text { Electron Energy } \\
\text { (Delayed Neutron } \\
\text { yield) }\end{array}$ \\
\hline "C". & $43.1 \pm 2.0$ & $6.2 \pm 0.1$ & $5.6 \pm 0.5$ & $\begin{array}{r}5.6+0.2 \\
-0.4\end{array}$ \\
\hline "8" & - & $\begin{array}{r}8.7+2.0 \\
-0.5\end{array}$ & $8.8 \pm 0.5$ & $8.6 \div 1.1$ \\
\hline
\end{tabular}

${ }^{a}$ Reference 2.

b References 4, 5, 6 .

electron beam current, the measured detector efficiency, and existing neutron yield measurements. $(4,5,6)$ The results are shown in Table 2; the uncertainty in the energy values assumes that the absolute calculated yields are correct to $\pm 50 \%$. Using all the available data, the calculated average electron energies are $5.8 \mathrm{MeV}$ and $8.7 \mathrm{MeV}$.

To check the stability and reproducibility of the electron energy spectrum, it is necessary to know the change in yield of the prompt and delayed $\mathrm{L}$.utrons for a given change in electron energy. Table 3 coniains these values as obtained from Ref. 2. The neutron yields rise steeply with increasing electron energy.

TABLE 3.

DEPENDENCE OF YIELD ON ELECTRON ENERGY

\begin{tabular}{|c|c|c|}
\hline \multirow{2}{*}{$\begin{array}{l}\text { Electron Energy } \\
\text { (MeV) }\end{array}$} & \multicolumn{2}{|c|}{$\%$ Change in Yield/Change in Electron Energy } \\
\hline & $\begin{array}{c}\text { Prompt Neutrons } \\
(\% / \mathrm{keV})\end{array}$ & $\begin{array}{l}\text { Delayed Netitrons } \\
(\% / \mathrm{keV})\end{array}$ \\
\hline 5.8 & 1.2 & 0.80 \\
\hline 8.7 & 0.04 & 0.07 \\
\hline
\end{tabular}


The yield is seen to be much more sensitive to a change in electron energy at the lower energy where the slope of the yield curve is steeper.

Table 4 shows the limits on stability and reproducibility as indicated by the data, using the sensitivities in Table 3 . In order to use the best statistical data, the prempt neutron yields were used at $5.8 \mathrm{MeV}$ and the delayed neutron yields at $8.7 \mathrm{MieV}$. The quantity $\Delta Y$ in Table 4 indicates the percentage change in yield; the quantity $\Delta \mathrm{E}$ indicates the corresponding calculated change in the median energy of the electron beam.

\section{TABLE 4.}

STABILITY AND REPRODUCIBILITY OF

ELECTRON ENERGY SPECTRUM REPRODUCIBILITY

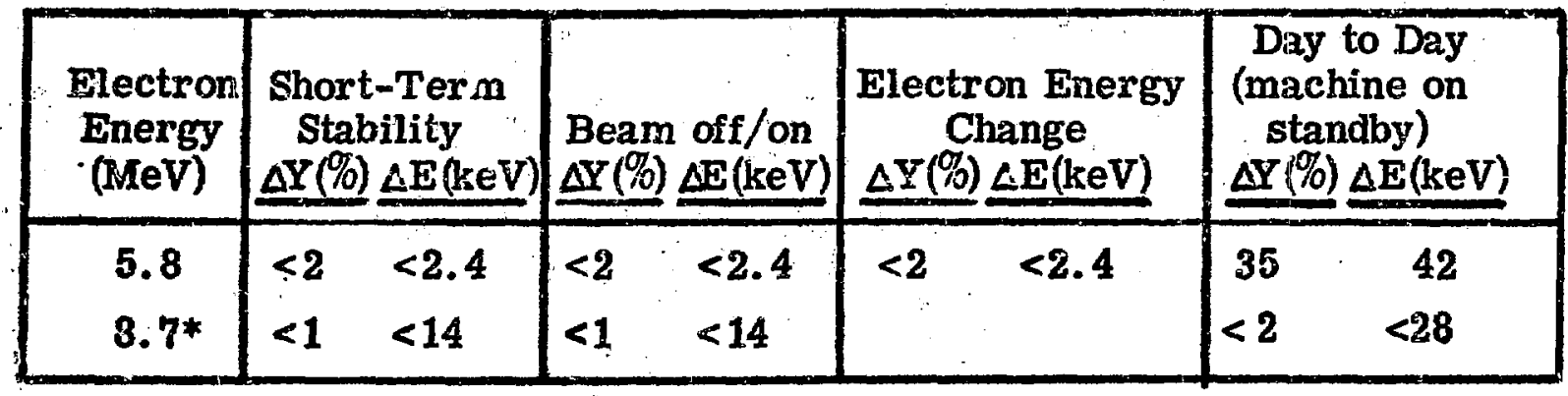

* The statistics on the delayed neutron yields at $8.7 \mathrm{MeV}$ were about $1 \%$; therefore, only upper limits could be placed on the energy stability at 3. $7 \mathrm{MeV}$.

Stability in the yield is defined as follows: the change in yield between successive runs of a few minutes length. Sets of runs were made to check the short term stability of the accelerator during a single nuclear materials assay which typically lasts 5 to 10 minutes. In the machine off/on test, the high voltage to the pulse forming networks and magnetron vas turned off for 2 minutes, the beam was turned on for 1 minute to build up the delayed neutron population, and then data runs were begun. This sequence corresponds to a set of typical assay runs for nuclear material where the sample is assayed for about 5 minutes, the accelerator is turned off to change samples for 1 minute, and then the operation is repeated. 
The electron energy change sequences are defined as follows: A series of machine off/on muns are made at $5.8 \mathrm{MeV}$. Next, the energy is switched to $8.7 \mathrm{MeV}$ and a second series of runs are made. The energy is then switched back to $5.8 \mathrm{MeV}$ for a third series of runs. This particular sequence corresponds to the situation where a set of assay samples are being assayed at two different electron energies to take advantage of the additional assay information which can be obtained by taking data at more than one electron energy.

The day-to-day reproducibility checks were performed on separate days with the machine left in standby overnight. In the standby mode the electron gun and magnetron filaments remain on at $1 / 4$ standby power, the thyratron is off, and the water pump is on. There is a built-in 5 minute delay when turning the machine on from the standby thode, which allows time for the thyratron, magnetron, and gun to come up to operating temperature. In normal assay operation, the machine would be left in the standby mode overnight (if it were not operating on a 24 hour basis) or during weekends.

\section{EVALUATION}

Stability and Reproducibility

The following comments on stability and reproducibility apply to the Varian standing wave, side coupled linac operating without a magnetic analysis system:

1. In order to be useful for assay work, the neutron yield per dose from a linac assay syatem must be stable over periods of a few minutes. This necessary condition for short term stability of neutron yield/dose is satisfied to within $2 \%$ at the lower end of the range of useful assay energies $(5-7 \mathrm{MeV})$ and by less than $1 \%$ at the upper end ( $7-9 \mathrm{MeV})$ over periods of $2-3$ minutes:

2. A more stringent assay condition which must also be satisfied is that the results from an assay must be reproducible after the accelerator beam has been turned off for a period of a few minutes. During the beam-off period, unknown samples can be exchanged or a standard sample can be substituted for an unknown sample. For the system 
under discussion, the yields for two assays as defined above vary by less than $2 \%(1 \%)$ in the lower (upper) end of the range of useful assay energies.

3. Although it is not always necessary, more information can be obtained about the contents of an unknown sample by assaying it at two electron energies rather than just one. The accelerator under discussion can be switched from one assay energy $\left(E_{1}\right)$ to another $\left(E_{2}\right)$ and back again to $E_{1}$ and the assays at energy $E_{1}$ will differ by less than $2 \%$. The variation in yield observed here, compared to the variation observed between assays at the same energy [ (2) above], is not particularly significant. If the situation demands that a number of unknown samples and standards be assayed at two different energies with maximum precision, the best procedure to follow would be to assay all the samples first at one energy, and then all of them at the second energy. With the system under discussion here, the operator could assay a second batch of samples together with his standards, and be assured or retuining close enough to his previous assay energy so that his standard yields would vary by less than $2 \%$ from those measured with the first batch of samples. In other words, the basic operating condition of the accelerator changes insignificantly between batches. Each batch of unknown samples measured within that batch, as a matter of operating procedure.

4. Assuming the accelerator is placed in a standby condition overnight, the operator can expect the observed yields from his standard sample to vary from day to day by less than $40 \%$ at the lower end of the useful range of electron energies and by less than $4 \%$ at the upper end of this range. These yield variations correspond to an equivalent electron energy change of less than $50 \mathrm{keV}$. Therefore, although the neutron yield/dose can change significantly from day to day, the equivalent electron ener only changes by 0.6 to $0.9 \%$. It is thus apparent that the basic setting of the machine does not change significantly from day to day. . (For example, 


\begin{abstract}
a system set up to assay samples at $6.5 \mathrm{MeV}$; below the lead $(y, n)$ threshold at $6.7 \mathrm{MeV}$, should not be expected to drift $200 \mathrm{keV}$ up in energy above the lead threshold from one day to the next.). The variation in neutron yield/dose between one day's operations and the next can be handled easily by remeasuring the standard samples at least once each day.
\end{abstract}

\title{
Operational Characteristics
}

The linac was very simple to operate; this feature was intentionally built into the design of the accelerator since it was destined for use in a radiotherapy treatment center. This fact is partially illustrated by the simple control panel for the system shown in Fig. 2. The accelerator would normally be operated by hospital technicians--personnel usually unfamiliar with the internal functions of the accelerators or their associated hardware.

The basic accelerator controls include:

1. Main power on/standby/off switch.

2. Beam on/off switch.

3. Pulse repetition rate control.

4. Frequency control--normal/automatic.*

5. Accelerator mode switch $(6,8$ or $10 \mathrm{MeV})$.

The accelerator is completely interlocised to provide for the safety of operating personnel.

The time necessary for the accelerator to become operational after making major changes in its operating mode are given in Table 5.

The 3 position mode switch is used to select any one of three electron energies and corresponding beam currents. The electron energy and beam current for any given mode can be varied (within the design limits

* The linac AFC (automatic frequeney control) circuitry was not operational during the tests described here. 
TABLE 5.

TIME REQUIRED FOR CHANGES IN

OPERATING MODES

\begin{tabular}{|l|l|}
\hline \multicolumn{1}{|c|}{ Change in Operational Mode } & Time Required \\
\hline Beam on from completely shutdown condition & Several hours * \\
Beam on from standby condition & five minutes \\
Beam on from beam off & Several seconds \\
Change electron energies & Several seconds \\
\hline
\end{tabular}

*This time is required to raise the various component temperatures to a steady state condition.

of the machine) by making internal adjustments to change the injected current and to change the RF power delivered to the waveguide.

For applications where a large number of identical or similar samples are to be assayed, several preselected modes could provide an adequate range of settings for the accelerator. This would provide for simple and easy operation of the device. (For exarnple, a fuel fabricator might find that two settings, one at $6.0 \mathrm{MeV}-60 \mu \mathrm{A}$, and another at $8.0 \mathrm{MeV}-1 \mu \mathrm{A}$ would be sufficient to assay initial and final products while a third mode of $7.0 \mathrm{MeV}-50 \mu \mathrm{A}$ wculd handle the scrap problem.) The different modes could also all be at approximately the same energy but with different beam currents. The number of modes available is not limited to three. * For applications such as these, no internal adjustments should be necessary during normal operation of the accelerator.

A second type of application concerns systems used in research and development laboratories and/or in government mobile assay laboratories. These applications require a large number of different operating modes tailored to a large number of diverse problems. It is unlikely that even a large number of discrete operating modes would cover all the conceivable

* Costs will be somewhat greater than the given estimates if more than three modes are required. 
situations. In these cases, personnel trained to make other internal final adjustments to several basic operating modes would provide the flexibility necessary to adequatèly handle most problems. Laboratories and other. similar facilities are usually staffed with highly skilled technical personnel anyway, so that the need for trained aceelerator personnel in these situations is not an unusual or awkward requirement.

The system tested was not equipped with an analyzing magnet. Analyzing magnets are used with linac systems for two reasons:

1. to determine the energy of the beam.

2. when used with analyzing slits or beam dumps, to improve the energy resolution of the electron beam and hence the stability of the assay system.

The results presented above show that this standing wave, side coupled linac has a sufficiently stable electron spectrum so as not to require a magnetic analysis system for increased system stability. This is a significant advantage over traveling wave accelerators which do require a magnetic analysis system and analyzing slits or beam dump. These systems use only part of the electron beam to perform the assay. The unused portion of the beam is stopped in analyzing slits or diverted into a beam dump. The radiation from the unused portion of the beam must be shielded to prevent it from interacting with the sample and also to protect operating personnel. This implies additional weight, size, cost and complexity for the system as compared to a standing wave linac which requires no additional analysis system.

On the other hand, the analyzing magnet is useful for precisely determining the energy of the electron beam. This may not be necessary or useful for a system which is normally required to operate in only several different modes (eg. in a fuel fabrication facility). It would be very useful for a laboratory set-up where good system fllexibility is required. The magnet system would not be used to perform assays, but only to initially set-up the machine at the electron energy desired. Therefore, all the accelerated beam would still be used to perform the assays. The magnet system could be constructed as an easily removable item and could be available on loan from the manufacturer for making changes in the normal operating modes of less flexible systems if they are required. 
An obviais detail which should not be overlooked in future Safeguards Inac systems is the choice of gamma shielding material. The present tests were run with a bremsstrahlung collimator constructed primarily of tungsten.* This collimator produced a large neutron background at energies above the lowest tungsten $(\gamma, n)$ threshold of $6.2 \mathrm{MeV}$. A better choice for gamma shielding would have been copper; neutrons are not emitted from copper for electron energies less than $9.9 \mathrm{MeV}$.

\section{SUMMARY}

The neutron yield/dose for a nuclear materials assay system employing a side coupled standing wave linac without a magnetic analysis system, is stable to within $2 \%$ at the lower end of the range of useful assay energies and to within $1 \%$ at the upper end. Assays separated by beamoff periods (during which samples can be exchanged) and by electron energy changes are reproducible to within $2 \%(1 \%)$, for the lower (upper) end of this same energy range. Day to day reproducibility of the yield is significantly less, however, the operating point of the system changes by less than $50 \mathrm{keV}$ from one day's operation to the next. These figures show the system to be usefui for most assay afplications.

The linac tested was simple to operate and was interlocked for radiation hazard safety. The times required to make major changes in the linac's operating mode (eg., eikergy changes, beam off-on, machine stanciby-on') are negligible. Energy changes are especially simple and quick to make when using any one of three pre-selected operational modes. This feature is valuable for applications which require only a few operational modes. Research applications which require a continuously variable energy and current must make additional fine internal adjustments after selecting a basic mode near the desired operating point.

Analyzing magnet systems are not required for stability and reproducibility. Unlike traveling wave linac systems, the entire electron beam can be utilized for assay purposes. However, systems wilich are used to assay a large variety of sample configurations (such as mobile assay urits or research facilities) should have a simple magnet system to determine the beam energy. This will allow rapid set-up of the system for new types of gamples and aiso save time during research in which one is trying to determine optimum operating points for different types of samples.

These slits were already installed on the accelerator that was tested and could not be easily removed. 


\section{ACKNOWLEDGEMENTS}

The authors would like to express their sincere thanks to the following additional personnel who took an active part in planning, preparing for and making these tests.

JRB Associates, La Jolla

J. R. Beyster

Stanford Nuclear Engineering Department

R. Sher

Technical Services Organization - BNL

W. Higinbotham

M. Zucker

Technische Universitat Hannover

D. Stegemann

Varian Associates, Palo Alto

D. Lawrence

B. Meyer

D. Penning

D. Reid

\section{REFERENCES}

1. J. R. Beyster, "Status of Commencial Electron Accelerators for Safeguards Applications," JRB 69-122 (1969).

2. Gulf General Atomic Report, GA-9077 (1968).

3. Gulf Radiation Technology Report, GA-10272 (1970).

4. Applied Radiation Corporation Report, AM-102 (1957). 
5. I. Katz, A. P. Baerg, F. Brown, Proceedings of the Second United Nations Conf. on Peacefal Uses of Atomic Erie:gy. Vol. 15, p. 188.

6. O. P. Nikotin, K. A. Petrzhak, Atomnaya Bnergiya 20 (1965) 268.

APPENDIX I: GENERAL SPECIFICATIONS FOR THE

VARIAN LINATRON 1500 STANDING WAVE, SIDE COUPLED ELECTRON LINEAR ACCELERATOR

Energy Range:

Current Available:

Pulse Width:

Pulse Repetition Rate:

Cooling Water Requirements:

Electrical Power Requirements:

Components:

$X$-ray Head:

29" height

28" width

66 "' depth

Modulator Cabinet: : 83" height

$48^{\prime \prime}$ width

30" depth

Control Console: $10 "$ height 20" width

15" depth
4-10 MeV

up to $110 \mathrm{ma}$ (peak)

$3-4 \mu \mathrm{sec}$

Continuously variable between

80 and 320 pps

$4 \mathrm{gpm}$ (machine on)

15 KVA

$2000 \mathrm{lbs}$.

1500 lbs.

$60 \mathrm{lbs}$.

Model Switch:

Three energy-beam current combinations can

Normal Price (1 unit): \$170, 000. be selected, using $a^{2}$ three position switch.

Normal Price (10 units): It appears possible to reduce the above ccst by $25-30 \%$. 